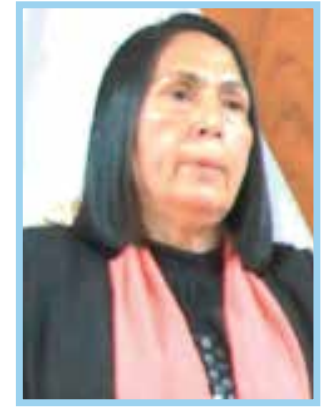

Yadira Jiménez

Arrunátegui

\section{ACREDITACIÓN: \\ UN PROCESO VITAL PARA LA \\ INTERNACIONALIZACIÓN \\ UNIVERSITARIA}

\section{RESUMEN}

Se presenta el referente histórico de la acreditación universitaria en la región de Latinoamérica, una realidad que se extiende y dinamiza, como una exigencia del aseguramiento de la calidad educativa basada en la enseñanza, investigación y mejores servicios, pertinencia de los planes de estudios, mayores posibilidades de empleo para los egresados, establecimiento de acuerdos de cooperación y la igualdad de acceso a los beneficios que reporta la cooperación internacional (CONEAU 2008).

\section{PALABRAS CLAVE}

Acreditación, universidad, calidad, internacionalización universitaria.

\section{ABSTRACT}

History reference of university accreditation is presented in Latinoamerica region, a reality expanding and putting energy into, as a demand of quality assurance education based on teaching, searching and better services, pertinence of study plans, greatest job chances for graduates, setting out of cooperation and equality access agreements the benefits brought by international cooperation (CONEAU 20I8).

\section{KEYWORDS}

Accreditation, university, quality, university internationalization. $\square$ n los países de América del Norte: Estados Unidos y Canadá, la acreditación universitaria surge entre finales del siglo XIX y XX principalmente sumada a la creación de agencias de escala nacional que evaluaban y acreditaban periódicamente los programas de formación profesional, mediante pares disciplinarios (García, 2010).

La evaluación y la acreditación de la calidad en la región latinoamericana se inician desde fines de los 90, convirtiéndose en una característica de los Sistemas de Educación Superior del mundo globalizado del siglo XXI.

El término calidad aplicado a la educación superior incluye los procesos internos de evaluación y mejora de la calidad, generados y desarrollados por las propias instituciones de educación superior que pretenden ser un medio de auto estudio analítico y reflexivo del desempeño de la Institución con el propósito de realizar los ajustes oportunos para impulsar y potenciar el trabajo académico y la Gestión Universitaria y, los procesos externos, que se promueven desde las autoridades de gobierno a través de diferentes instrumentos (legislación, agencias de aseguramiento de la calidad), con el objetivo de verificar que una institución cumple con las características y estándares de calidad de tal manera que pueda certificar ante la sociedad la calidad académica y la integridad institucional y así ser certificada o acreditada.

La calidad de la enseñanza superior es un concepto pluridimensional, que involucra a docentes y estudiantes, en tanto actores de programas académicos, procesos pedagógicos 
y de proyectos de investigación, buscando poner en común los conocimientos y lograr mejores estándares de calidad, tanto para responder a las oportunidades y a las nuevas perspectivas de la educación superior, como para enfrentar una serie de desafíos y dificultades, como producto del entorno cambiante que conlleva la globalización.

"La dimensión internacional de la educación superior es un elemento intrínseco de su calidad. El establecimiento de redes, que ha resultado ser uno de los principales medios de acción actuales, ha de estar fundado en la ayuda mutua, la solidaridad y la igualdad entre asociados.... Ha de darse prioridad a programas de formación en los países en desarrollo, en centros de excelencia organizados en redes regionales e internacionales, acompañados de cursillos en el extranjero especializados e intensivos de corta duración" (Conferencia Mundial sobre la Educación Superior, 1998).

Como producto de la globalización, el conocimiento se convierte en un elemento transable, al amparo de los acuerdos de libre comercio, como el GATS y el ALCA que buscan liberalizar los servicios de enseñanza y han convertido a la educación en una industria que mueve millones de estudiantes y genera billones de dólares anualmente, transformando este sector educativo en una fuente infinita de riqueza, con la generación de nuevas formas de llevar la educación, como las franquicias, los jointventures y las licencias, las cuales se perciben hoy en día como la "nueva internacionalización" (ASCUM, 2003, p. 39).

En contraposición a lo señalado, un estudio del Instituto Internacional para la Educación Superior en América Latina y el Caribe (IESALC) de la UNESCO caracteriza a la educación superior tanto en el plano local como en el plano global.

En el plano local caracterizado por restricciones financieras de las universidades públicas; sistemas de limitación en el acceso; nuevas formas de regulación a través de Consejos de Rectores, con diferenciación institucional y mayor presencia en número de instituciones privadas; alta feminización de la matrícula estudiantil; creciente desempleo y emigración de profesionales y técnicos; nuevas demandas de habilidades y destrezas en los mercados laborales; propagación de actividades y creación de organismos para la evaluación y la acreditación de programas, departamentos, instituciones, docentes y estudiantes.

Respecto del plano global, la educación superior se caracteriza por una creciente internacionalización, el establecimiento de alianzas; la incorporación de la educación virtual; la convergencia digital de industrias culturales y servicios educacionales; el interés por desarrollo del conocimiento científico y nacimiento de una economía de redes (Siufi, 2008).

"Si la globalización es el fenómeno, la internacionalización de la educación superior es una de las respuestas a este suceso, manifiesta en un proceso que es determinante para alcanzar su inserción en el mundo del conocimiento como una de las formas de desafiar los retos que se le imponen" (Asociación Colombiana de Universidades, ASCUM, 2003, p. 4).

La suscripción de la Declaración de la Sorbona (1998) y la Declaración de Bolonia el año siguiente (1999), facilitaron el desarrollo de criterios y metodologías comparables en la educación superior universitaria, lo que ha sido propicio para crear condiciones que facilitaron el desarrollo de la internacionalización universitaria.

Entre estas condiciones se desarrolla el Espacio de América Latina y del Caribe-Unión Europea (ALCUE), el Espacio Iberoamericano del Conocimiento (CINDA, 2007) y la iniciativa IESALC-UNESCO 2008.

Así también, por medio del proyecto ENLACES (ENLACES, 2009), con nuevos programas como 
TEMPUS con la Europa del Este y la cuenca mediterránea, ALFA con América Latina, ATLANTIS con Estados Unidos y recientemente ERASMUS MUNDUS en el marco de programas conjuntos de master o doctorado.

La Declaración de Bolonia firmada por 29 ministros de educación europeos incluía entre sus objetivos la formación de un sistema fácilmente legible y comparable de titulaciones; se promovía también, entre otros, la cooperación para asegurar un nivel de calidad para el desarrollo de criterios y metodologías comparables, lo que no siempre fue fácil, pues los países tenían y tienen sistemas de educación superior muy diversos.

Los programas de la Unión Europea: ALBAN, Erasmus Mundus y ALFA, entre otros, fueron creados para convertir a Europa en el principal destino para realización de estudios superiores para países terceros y en el cooperante más importante en materia de educación.

Estos programas han tenido, en la Unión Europea desde la década del ochenta, con el Programa ERASMUS, el modelo de programa para facilitar la integración de sus miembros, fue la principal herramienta de promoción de la movilidad de docentes, investigadores y estudiantes, aun cuando en su inicio no fuera un objetivo de las universidades la movilidad universitaria sino la convergencia en investigación. En lo que respecta el Programa ALBAN, instaurado desde 2002 y que se prolongara hasta 2010, se realizaron cinco convocatorias, que resultaron en 3319 becas para candidatos de 18 países de América Latina, para realizar estudios de Maestría o Doctorado (Da Silva, 2011).

En el caso de los países de América Latina se vive una doble dimensión del proceso de internacionalización: uno regional y otro mundial. En el marco regional, el proceso se relaciona con la siempre ansiada integración latinoamericana, objetivo que contempla razones muy profundas que tienen que ver con ideales de unión cultural y espiritual de los pueblos. En la escala planetaria, el proceso se relaciona a la necesidad de aumentar la calidad, la productividad científica y a promover una mayor competitividad de la Región (Proyecto Safiro UE).

Los procesos de internacionalización de las instituciones de educación superior se concretan y materializan mediante distintas estrategias y acciones que las universidades deciden desarrollar, entre las cuales se pueden enumerar las siguientes: movilidad estudiantil, movilidad de profesores e investigadores, participación en redes de carácter regional e internacional, oferta educativa internacional (educación trasnacional), titulaciones conjuntas con instituciones extranjeras, acuerdos interinstitucionales (convenios generales y específicos), investigaciones conjuntas con grupos extranjeros, oferta de enseñanza de idiomas y cultura locales, acciones de cooperación al desarrollo, internacionalización del currículum.

En el Perú, con la promulgación de la Ley № 28740 (ley del SINEACE, 2006), se inicia el camino a la acreditación de la calidad de las instituciones educativas y de sus programas; que además aprueba la creación del Consejo Nacional de Evaluación, Acreditación, Certificación de la Calidad de la Educación Universitaria CONEAU como instancia competente para la acreditación de las universidades y sus carreras profesionales y programas de posgrado.

En el Modelo de Calidad del CONEAU del Perú se identifican tres factores, uno de los cuales el factor enseñanza - aprendizaje, incluye acciones de mejora a los estudiantes, referidas a los medios que se les ofrece para su mejor desempeño intelectual, académico y profesional, vinculados con la oferta y demanda de estudios en el extranjero a través de la movilidad universitaria, como indicador de la calidad del servicio en los programas de formación profesional universitaria. 
En la Universidad Femenina del Sagrado Corazón (UNIFÉ), la experiencia inicial ha sido la movilidad de docentes para la enseñanza de idiomas en el Programa Académico de Traducción, la suscripción de convenios de colaboración académica y de investigación y la visita de profesionales extranjeros para el desarrollo de actividades de académicas.

En el quinquenio 2009 - 2013, la UNIFÉ ha enfatizado en la necesidad de promover su participación en diversos programas y mecanismos de cooperación internacional y hacer viables oportunidades para las estudiantes, como respuesta a nuevos retos, adquirir experiencia o establecer contactos con personas e instituciones que mejoren su desempeño profesional.

\section{REFERENCIAS}

Asociación Colombiana de Universidades (ASCUN) (2003). Agenda, Hacia una Internacionalización de la Universidad Con Sentido Propio. Bogotá, D. C. Recuperado de: http://secretariageneral.univalle. edu.co/consejoacademico/temasdediscusion/2003/ Agenda\%20de\%20Pol\%EDticas.pdf

Conferencia Mundial sobre la Educación Superior (1998). La Educación Superior en el siglo XXI. Visión y acción. París: UNESCO. Recuperado de: http://www.unesco.org/ education/educprog/wche/declaration_spa.htm

Da Silva, G. (2009). Internacionalización de la Educación Superior y movilidad estudiantil Universidad del Norte. Colombia. Recuperado de: http://politicacolombiana. net/pdfs/ledicion/articulo013.pdf
En este contexto, el año 201l, de acuerdo a lo señalado en los Estatutos de la UNIFÉ, se crea la Comisión Ejecutiva de Cooperación y Relaciones Internacionales (CECRI) para hacer evidente estas líneas de acción y promover su institucionalización.

A partir del año 2012, como participante del Programa de Movilidad Estudiantil de la Unión de Universidades Latinoamericanas (PAMEUDUAL), se ha recibido estudiantes de Brasil, México y Colombia; además, por Convenio entre centros de formación universitaria en la especialidad de Psicología, se reciben estudiantes de Inglaterra para su práctica comunitaria. Con una escasa demanda de las estudiantes de UNIFÉ por esta experiencia formativa en instituciones universitarias del extranjero.

Delgado-Márquez, B. (2011). La internacionalización en la enseñanza superior: investigación teórica y empírica sobre su influencia en las clasificaciones de las instituciones universitarias. Revista de Universidady Sociedad del Conocimiento, 8 (2) 101-122. Recuperado de: http://www.redalyc.org/articulo.oa?id=78018793009

Fasio, M. (2011). Internacionalización de los sistemas de evaluación y acreditación universitaria en América Latina (1998-2009). Meta evaluación de los sistemas desde la perspectiva comparada. Sociedad Argentina de Estudios Comparados IV Congreso. Recuperado de http:// www.saece.org.ar/docs/congreso4/trab43.pdf 
Figueroa, R. (2012). La autoevaluación institucional y su importancia en la educación superior. Humanidades Médicas, 12 (3), 447-463. Recuperado de http://www. humanidadesmedicas.sld.cu/index.php/hm/article/ view/254/162

Franco, J.; Piva, H.; Riccomi L. y Singer, A. (2011). Losprocesos de acreditación regional de carreras de grado (MEXA/ARCUSUR). La educación Superior como pilar para la integraciónyel desarrollolatinoamericano. I Congreso Red de Integración Latinoamericana. REDILA. Recuperado de http:// www.uncu.edu.ar/relacionesinternacionales/upload/ redilaejel3.pdf

Gacel-Ávila, J. (1999). Internacionalización de la Educación Superior en América Latina y el Caribe. Reflexiones y Lineamientos. Guadalajara, México: Organización Universitaria Interamericana.

García, Ó. A. (compilador) (2003). Hacia una nueva universidad en el Perú. Lima, Perú: UNMSM, Fondo Editorial.

LLaque, L. (2005). Estudio de la internacionalización de nuevos proveedores en la Educación Superior en el Perú. Lima, Perú: Asamblea Nacional de Rectores.

Larrea, Marina B.; Astur, Anahí M. (2012). Políticas de internacionalización de la educación superior y cooperación internacional universitaria. Consultado en http://portales. educacion.gov.ar/spu/files/2011/12/Art\%C3\%ADculoPol\%C3\%ADticas-de-internacionalizaci\%C3\%B3nde-la-ES.pdf

López I. (2004). La acreditación en el Perú. Revista Iberoamericana de Educación. OEI. Recuperado de http:// rieoei.org/rie35a06.pdf
Ministerio de Educación (2004). Informe sobre la Educación Peruana. Recuperado de http://www.oei.es/quipu/ peru/ibeperu.pdf

Moctezuma, P.; Navarro A. (2011). Internacionalización de la educación superior: aprendizaje institucional en Baja California. Revista de la Educación Superior, Vol. XL (3), № 159, pp. 47-66. Recuperado de http://www. redalyc.org/pdf/604/60422576003.pdf

Monroy, D. (2008). Internacionalización de las IES en México: un estudio por comparación de casos del Proyecto ALFA - Tuning (Tesis de Maestría). México: FLACSO.

Nava, H. (2003). Evaluación y acreditación de la educación superior. El caso de Perú. IESALC/UNESCO. Recuperado de http://dide.minedu.gob.pe/ xmlui/bitstream/handle/123456789/286/156.\%20 Evaluaci\%c3\%b3n\%20y\%20acreditaci\%c3\%b3n\%20 de $\% 201$ a $\% 20$ educaci\%c3\%b3n\%20superior. $\% 20$ El\%20caso\%20del\%20Per\%c3\%ba.pdf? sequence=1

Sebastián, J. (2004). Cooperación e internacionalización de las universidades. Buenos Aires, Argentina: Biblos.

Siufi, G. (2008). Cooperación Internacionale Internacionalización de la Educación Superior. Recuperado de www.unesco. org.ve/dmdocuments/biblioteca/libros/capitulos/.../7. pdf

Wit de H.; Jaramillo, I.; Gacel-Ávila, J.; Knight, J. (2005). Educación Superior en América Latina. La dimensión internacional. Bogotá: Banco Mundial. 